\title{
Phototherapeutic keratectomy for the treatment of dense subepithelial infiltrates after epidemic keratoconjunctivitis
}

\author{
Ioannis A. Mallias, Panagiota Mylova, Aikaterini Mouzaka, Anastasia Tasiopoulou, Ronis Christidis
}

Laser Plus Eye, Nea Smyrni, Athens, Greece

\begin{abstract}
This is a case report of a patient who developed dense subepithelial corneal infiltrates after epidemic keratoconjunctivitis and was treated with phototherapeutic keratectomy (PTK). The purpose of this case report is to explain the surgical technique and the results of PTK procedure.
\end{abstract}

KEY WORDS: epidemic keratoconjunctivitis, phototherapeutic keratectomy, PTK, dense subepithelial infiltrates

Ophthalmol J 2016; Vol. 1, No. 4, 147-150

\section{INTRODUCTION}

Epidemic keratoconjunctivitis (EKC) is a highly contagious viral conjunctivitis [1]. It is caused by a group of viruses known as adenoviruses that, in addition to infections of the ocular surface, are responsible for causing infectious diseases of the gastrointestinal tract and respiratory system illnesses such as the common cold virus. Some ocular signs/symptoms [2] are: conjunctival hyperaemia, erythema (redness) of bulbar and palpebral conjunctiva, ocular itchiness and irritation, chemosis (conjunctival oedema), photophobia, epiphora (excessive tearing), foreign body sensation, blurred vision, loss of visual acuity, eyelid swelling, follicular reaction, and epithelial keratitis. Some systemic symptoms that may occur are: lymphadenopathy, fever, headache, and fatigue. Usually the symptoms progress to the other eye, although they may be milder. Some patients develop persistent Subepithelial opacities.

Epidemic keratoconjunctivitis [3] usually resolves on its own. Many different types of treatments have been used with variable results. Antiviral medications have not been shown to be effective against viral conjunctivitis. Topical corticosteroids are often prescribed in severe cases although their use is controversial. A person can be contagious [2] after the beginning of symptoms.

\section{CASE REPORT}

A 50-year-old woman presented with severe decrease of visual acuity of the right eye. A thorough slit-lamp examination was performed by Dr. Mallias, which revealed severe subepithelial opacities in the right eye, as can be seen in Figure 1. The subepithelial opacities are also clearly shown in the OCT image (Fig. 2). The patient reported that her vision decreased one month after the onset of epidemic keratoconjunctivitis. Visual acuity was 20/200. The intraocular pressure was within normal limits.

A transepithelial phototherapeutic keratectomy (PTK) was performed by Dr. Mallias. An Excimer Laser Amaris 750s (Schwind, Germany) was employed in order to resolve the corneal opacities. The 


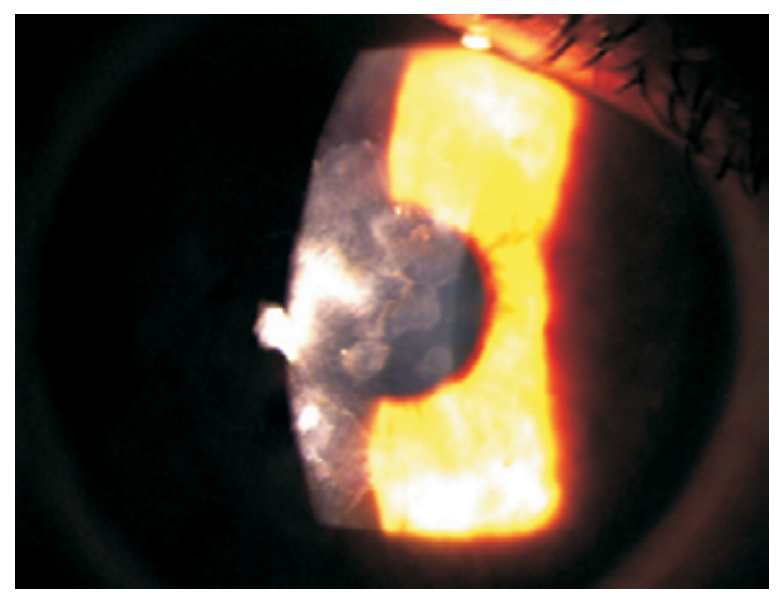

FIGURE 1. Preoperative slit lamp image of the patient

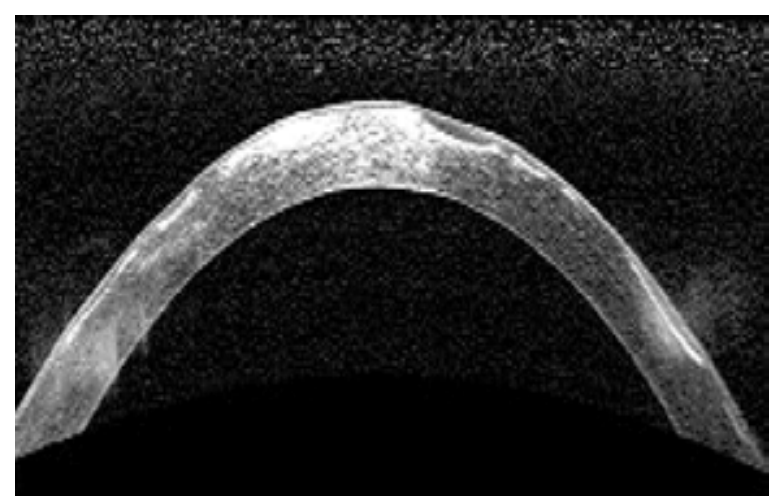

FIGURE 2. Preoperative OCT image of the patient first step of the surgery was the epithelium removal. In this case, the epithelium was removed by the Excimer Laser (transepithelial PTK). The next step was the application of a masking agent (Vismed Gel, AngeliniPharma) in order to smoothen the irregularity of the anterior corneal surface. Mitomycin C (MMC) $0.02 \%$ was used in order to avoid corneal haze. Dr. Mallias applied the MMC $0.02 \%$ with a small sponge in the cornea for 30 seconds and then rinsed thoroughly with a balanced salt solution (BSS). After the end of the operation, a bandage soft contact lens was applied until epithelial healing was achieved.

The ablation depth was $120 \mu \mathrm{m}$, the ablation zone was $8.50 \mathrm{~mm}$, and the ablation time was 45 seconds. More information about the ablation is displayed in Figure 3, which depicts the ablation profile. Minimum corneal thickness was $372 \mu \mathrm{m}$ preoperatively and $335 \mu \mathrm{m}$ postoperatively.

Loteprednol was prescribed to the patient four times daily for three weeks and was slowly tapered over a period of three months. She was also prescribed ofloxacin to use for five days in order to avoid infection as well as artificial tears to use for at least two months. Epithelial healing was achieved five days postoperatively and the contact lens was removed. Visual acuity improved from 20/200 to

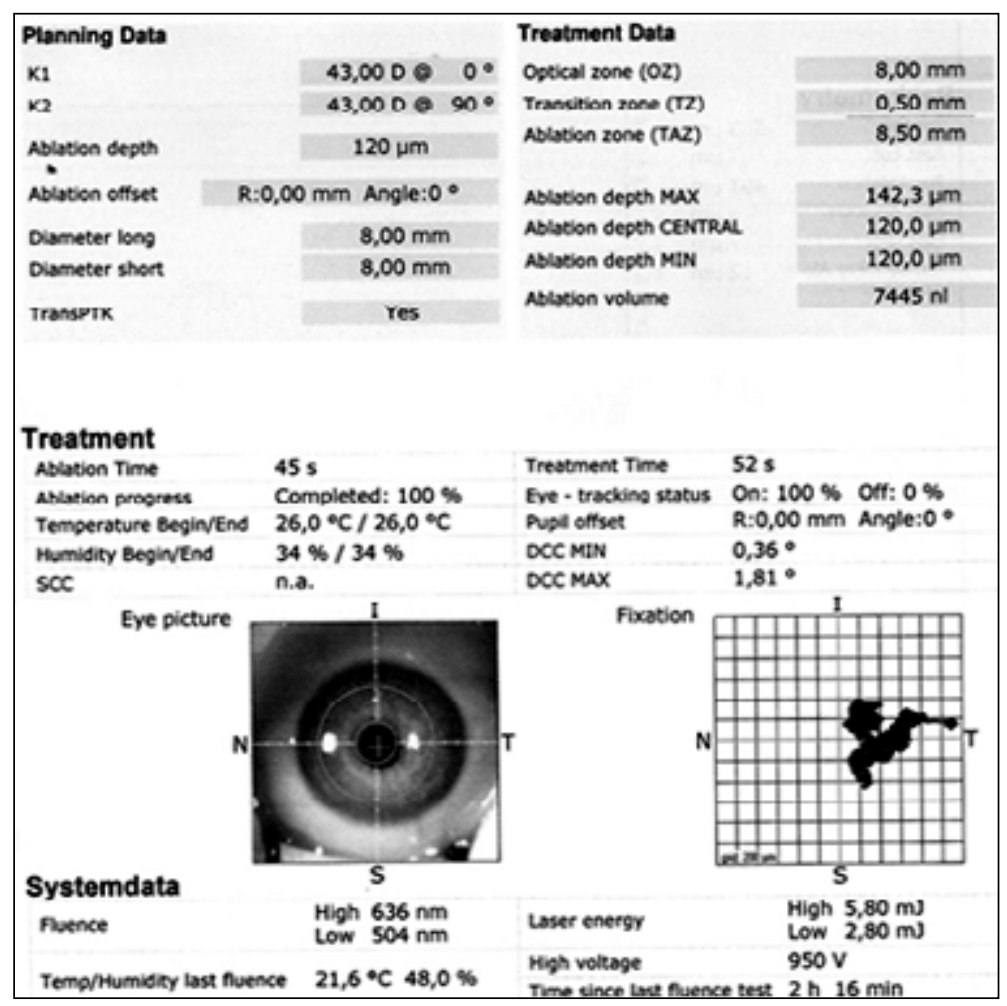

FIGURE 3. Ablation profile 


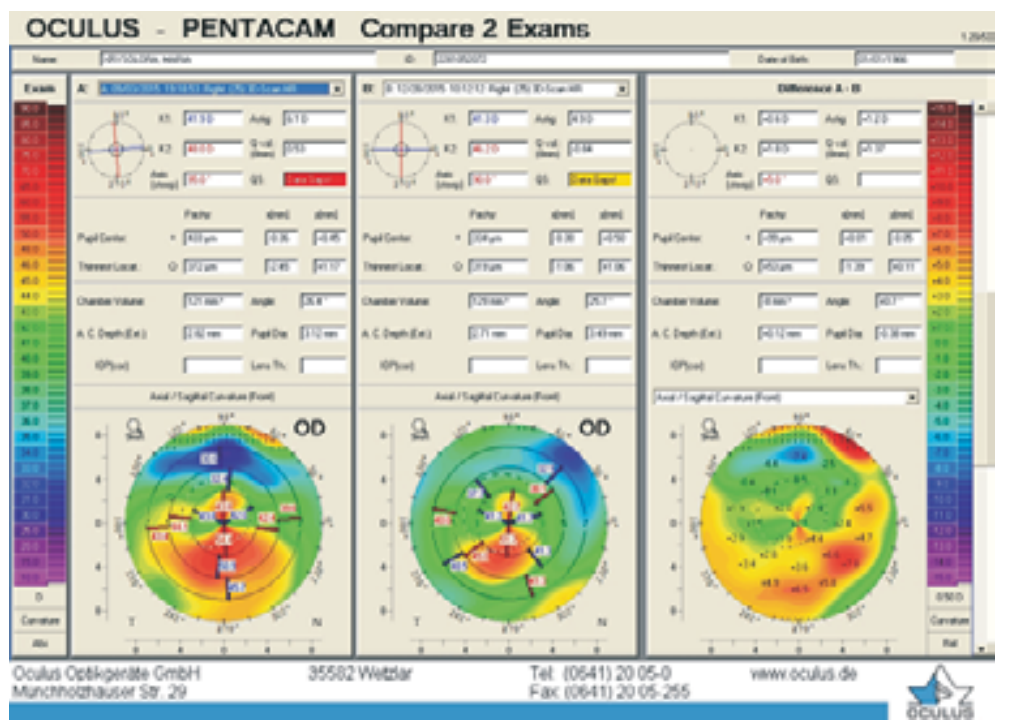

FIGURE 4. Pre- and postoperative topographies of the patient and comparison of them

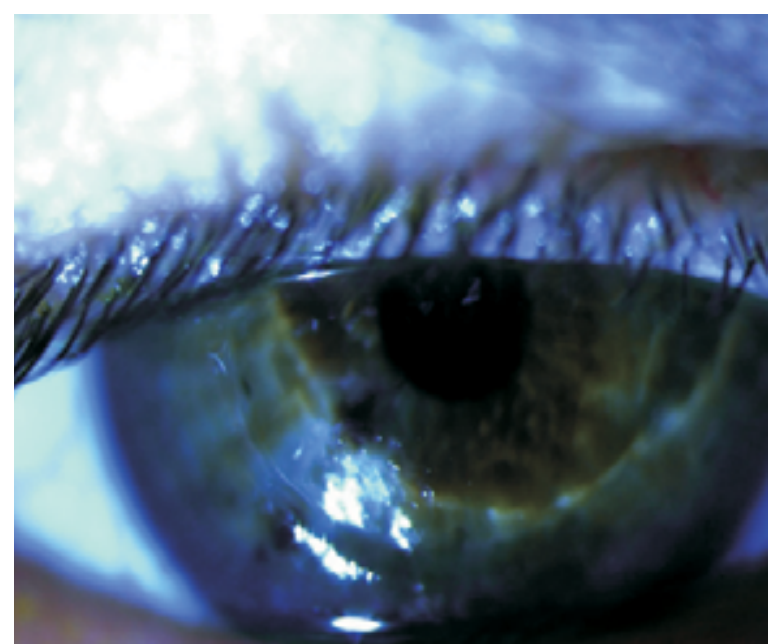

FIGURE 5. Postoperative slit lamp image of the patient

20/100 one month after the procedure. One year after surgery the patient has Best Spectacle Corrected Visual Acuity 20/40 and is very pleased with the outcome. The cornea is "smoothened" from the procedure and has become less irregular, as shown in the Pentacam images (Fig. 4). Also, there is no sign of corneal infiltrates or haziness in the anterior stroma, as can clearly be seen in Figure 5.

\section{DISCUSSION}

A common problem after Epidemic keratoconjunctivitis is the development of subepithelial infiltrates [4]. The first eye that is involved has more inflammation and develops more infiltrates than the second eye. Usually the Subepithelial infiltrates fade with steroid drops, although a common side effect of them is the intraocular pressure elevation. The prolonged use of steroid drops also increases the risk of cataract formation. There are some reports that Subepithelial infiltrates decrease with the use of topical cyclosporine $0.05 \%$ [5]. Our personal experience is that it works only in a small percentage of cases.

In this particular patient, we prescribed steroids for a month after initial presentation, but the infiltrates did not subside. We would like to stress the fact that our patient had an unusual presentation of subepithelial infiltrates after EKC. Usually we see small, dot-like subepithelial infiltrates, but this particular patient had large subepithelial infiltrates with scarring. We believe that this is the reason that the patient did not show any improvement with the use of steroids. In order to rehabilitate the patient's vision, we had to choose between the following options: 1) PTK, 2) anterior lamellar corneal transplant. In order to avoid anterior lamellar corneal transplant, we proceeded with the PTK surgery. There are also some studies $[6,7]$ referring to treatment of subepithelial infiltrates after EKC with PTK or even with PRK (Photorefractive Keratectomy) in order to diminish the refractive error as well.

What has come to our attention is that after performing PTK, these eyes appear to make a hyperopic shift [8]. This happens mainly due to the ablation depth, and it can be prevented by enlarging the treatment zone and using a masking agent during the phototherapeutic keratectomy. Our treatment zone in this patient was $8 \mathrm{~mm}$. There are studies [9] 
that indicate that making PTK a two-step procedure can result in a shallower ablation and as a result in a lower hyperopic shift.

Something that needs to be investigated further is the fact that some patients after epidemic keratoconjunctivitis develop large subepithelial infiltrates with scarring instead of dots, as happened in this case. These occur due to a more intense inflammatory reaction, which is probably due to pre-existing blepharitis. It is well known that in patients with meibomian gland disease the levels of various inflammatory agents rise, especially metalloproteinase 9 (MMP9), and maybe this is the reason for higher rates of haze formation in people who suffer from blepharitis.

\section{REFERENCES}

1. Adenovirus-Associated epidemic keratoconjunctivitis outbreaks — four states, 2008-2010. Morbidity and Mortality Weekly Report
2013, 62: 637-641. http://www.cdc.gov/mmwr/preview/mmwrhtml/ mm6232a1.htm.

2. http://eyewiki.aao.org/Epidemic_Keratoconjunctivitis.

3. Bawazeer A. Epidemic keratoconjunctivitis. medscape. http://emedicine.medscape.com/article/1192751-overview\#a0101.

4. Epidemic keratoconjunctivitis: A review of current concepts in management. Journal of Optometry 2013; 6. http://www.journalofoptometry. org/en/epidemic keratoconjunctivitis-a-review-of/articulo/90197282/.

5. Cakmak H, Ozbagcivan M, Kocaturk T et al. Effectiveness of $0.05 \%$ cyclosporine in the treatment of subepithelial infiltrates related with adenoviral keratoconjunctivitis. J Clin Case Rep 2013; 3: 4. http:// dx.doi.org/10.4172/2165-7920.1000268.

6. Yamazaki ES, Ferraz CA, Hazarbassanov RM, Allemann N, Campos M. Phototherapeutic keratectomy for the treatment of corneal opacities after epidemic keratoconjunctivitis. Am J Ophthalmol 2011; 151: 35.e1-43.e1.

7. Alevi D, Barsam A, Kruh J, Prince J, Perry HD, Donnenfeld ED. Photorefractive keratectomy with mitomycin-C for the combined treatment of myopia and subepithelial infiltrates after epidemic keratoconjunctivitis. J Cataract Refract Surg 2012; 38: 1028-1033.

8. Dogru M, Katakami C, Yamanaka A. Refractive changes after excimer laser phototherapeutic keratectomy. J Cataract Refract Surg 2001; 27: 686-692.

9. Amm M, Duncker Gl. Refractive changes after phototherapeutic keratectomy. J Cataract Refract Surg 1997; 23: 839-844. 